\title{
On finite group actions on reductive groups and buildings
}

\author{
Gopal Prasad $^{1, \star}$, Jiu-Kang Yu ${ }^{2, \star \star}$ \\ 1 Department of Mathematics, University of Michigan, Ann Arbor, MI 48109, USA \\ (e-mail: gprasademath. Isa.umich.edu) \\ 2 Department of Mathematics, University of Maryland, College Park, MD 20742, USA \\ (e-mail: yu@math. umd. edu)
}

Oblatum 8-III-2001 \& 25-VII-2001

Published online: 19 November 2001 - (C) Springer-Verlag 2001

Dedicated to Jacques Tits

\section{Introduction}

Let $H$ be a connected reductive group over a non-archimedean local field $k$ and let $F \subset \operatorname{Aut}_{k}(H)$ be a finite group of order not divisible by $p$, the residual characteristic of $k$. Let $G=\left(H^{F}\right)^{\circ}$ be the identity component of the subgroup of $H$ consisting of points fixed by $F$. The main theorem of this paper asserts that the Bruhat-Tits building $\mathscr{B}(G)$ of $G$ can be identified with the set of $F$-fixed points of $\mathcal{B}(H)$.

Several special cases of this theorem have been known previously. When $E / k$ is a finite totally ramified Galois extension, $H=\operatorname{Res}_{E / k} G$, and $F=$ $\operatorname{Gal}(E / k)$, the condition $p \nmid \# F$ is simply that $E / k$ is tamely ramified. In this case, our main theorem is a well-known but unpublished theorem of G. Rousseau. Recently, one of us (G.P.) found a simple proof of this theorem $[\mathrm{P}]$.

When $G$ is a classical group, realized in the standard way as the identity component of the group of fixed points of an involution $\phi$ of a general linear group $H$, Bruhat and Tits [BT4] have given a description of $\mathscr{B}(G)$ as a subset of $\mathscr{B}(H)$. In particular, it follows from their description that $\mathscr{B}(G)=$ $\mathscr{B}(H)^{\phi}$ when $p \neq 2$. Recently, this fact has been rediscovered by J. Kim and A. Moy [KM], and independently, a simple proof has been given by [GY].

Let $H$ be the split form of $\operatorname{Spin}(8)$. Then one can choose an outer automorphism $\phi \in \operatorname{Aut}(H)$ of order 3 such that $G=H^{\phi}$ is of type $G_{2}$. From a joint work [GY] by W.T. Gan and one of us (J.Y.), it is known that $\mathscr{B}(G)=\mathscr{B}(H)^{\phi}$ is true for arbitrary $p$.

* Partially supported by a Guggenheim Foundation Fellowship and an NSF grant

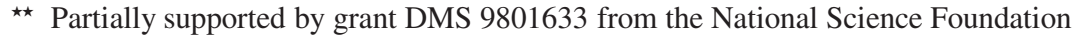


When $p \mid \# F$, in general $\mathscr{B}(H)^{F}$ may be strictly larger than $\mathcal{B}(G)$. See, for example, [T, 2.6.1].

The condition $p \nmid \# F$ implies that $\# F$ is not divisible by the characteristic of $k$, which ensures that $G=\left(H^{F}\right)^{\circ}$ is reductive. This result seems to be new, and two proofs are given in $\S 2$.

Similar results also hold for spherical buildings, and for symmetric spaces associated to real reductive groups. These phenomena prompted us to study the case of the Bruhat-Tits buildings. We will discuss the case of spherical buildings in $\S 3$ of this paper. The case of symmetric spaces is treated in an appendix to [GY].

Discussions with Wee Teck Gan and Dipendra Prasad are gratefully acknowledged. We would like to thank Anne-Marie Aubert, Stephen DeBacker, Bernhard Muehlherr and Richard Pink for their interest in this work. Part of this work was done while G.P. visited Forschungsinstitut für Mathematik, E.T.H., Zurich. He would like to thank the institute and its director Marc Burger for their hospitality and support.

\section{Notation}

- $k$ : a henselian field with respect to a discrete valuation ord.

$-\mathcal{O}$ : the ring of integers in $k$.

$-\mathfrak{p}$ : the prime ideal of $\mathcal{O}$.

- $\kappa$ : the residue field of $\mathcal{O}$. We assume that $\kappa$ is perfect of characteristic $p$.

- $H$ : a connected reductive group over $k$. We denote by $Z(H)$ the center of $H$.

- F: a finite subgroup of $\operatorname{Aut}_{k}(H)$.

- $G$ : the identity component of $H^{F}$, the subgroup of points fixed by $F$.

- $\mathcal{B}(H)$ : the (extended) Bruhat-Tits building of $H$ over $k$.

- For any torus $S$ over $k: S(k)_{\mathrm{b}}$ is the maximal bounded subgroup of $S(k)$, $X_{*}(S)$ is the set of one-parameter subgroups defined over $k$, and $V(S)$ is the real vector space $X_{*}(S) \otimes_{\mathbb{Z}} \mathbb{R}$.

- For any maximal $k$-split torus $T$ of $H: A(H, T)$ is the apartment in $\mathscr{B}(H)$ corresponding to $T$.

For $y \in \mathscr{B}(H)$ :

- $\underline{H}_{y}$ : the Bruhat-Tits group scheme over $\mathcal{O}$ attached to $y$ characterized by: (1) the generic fiber of $\underline{H}_{y}$ is $H$, (2) $\underline{H}_{y}$ is connected, smooth, and of finite type, (3) $\underline{H}_{y}\left(\mathcal{O}_{K}\right)$ is the parahoric subgroup of $G(K)$ associated to $y$, where $K$ is the maximal unramified extension of $k$ and $\mathcal{O}_{K}$ the ring of integers in $K$.

- $\bar{H}_{y}^{\text {red }}$ : the maximal reductive quotient of the special fiber of $\underline{H}_{y}$.

- For a parahoric subgroup $P=\underline{H}_{y}(\mathcal{O})$ of $H(k), P^{+}$will denote the kernel of the reduction map $P \rightarrow \bar{H}_{y}^{\text {red }}(\kappa)$.

- The notations introduced above for $H$ apply to $G$ and other connected reductive groups over $k$ as well. 
By a pro- $p$-group, we mean a group which is the projective limit of a projective system of groups whose members are iterated extensions of $\mathbb{F}_{p}$-vector spaces when $p>0$, or $\mathbb{Q}$-vector spaces when $p=0$. Thus $P^{+}$is simply the maximal normal pro- $p$-subgroup of $P=\underline{H}_{y}(\mathcal{O})$, and $H^{1}(F, J)=0$ whenever $J$ is a pro- $p$-group, $F$ is a finite group of order not divisible by $p$, and $F$ acts on $J$.

\section{The main theorem}

It will be shown in the next section that $G$ is a reductive group. We assume this fact in this section. Throughout this section, we assume that $\# F$ is not divisible by the characteristic $p$ of the residue field $\kappa$.

Until (1.9), we assume that $k$ is complete and strictly henselian (i.e. $\kappa$ is separably closed, and hence, as it has been assumed to be perfect, it is algebraically closed).

(1.1) Proposition Let $x \in \mathscr{B}(H)^{F}$ be a point fixed by $F$. Then there exist $k$-split tori $S \subset T$ such that

(i) $T$ is a maximal $k$-split torus of $H$ and $x$ lies on the corresponding apartment $A(H, T)$;

(ii) $S$ is $F$-stable, and the connected Néron model of $S$ reduces to the central torus $Z\left(\bar{H}_{x}^{\mathrm{red}}\right)^{\circ}$ of $\bar{H}_{x}^{\mathrm{red}}$.

Proof. Let $\mathcal{T}$ be the set of maximal $k$-split tori $T$ of $H$ such $x \in A(H, T)$. Then the finite group $F$ permutes $\mathcal{T}$ and the parahoric subgroup $P=\underline{H}_{x}(\mathcal{O})$ acts transitively on $\mathcal{T}$. For $T \in \mathcal{T}$, let $S=S_{T}$ be the lift in $T$ of the central torus of $\bar{H}_{x}^{\text {red }}$. It is clear that the pair $(S, T)$ satisfy (i) and (ii) as long as $S$ is $F$-stable. We will consider $\&=\left\{S_{T} \mid T \in \mathcal{T}\right\}$ and construct an $F$-stable element of $\&$.

Let $M_{S}$ be the centralizer of $S$ in $H$. Then $M_{S}$ is a Levi subgroup of $H$ containing $T, P \cap M_{S}$ is a maximal parahoric subgroup of $M_{S}$, and $P=\left(P \cap M_{S}\right) \cdot P^{+}$(see [MP, Prop. 6.4]). Let $P_{r}:=H_{x, r}$ be the "MoyPrasad" filtration ([MP]) on $P=H_{x}$ associated to the point $x$. Let $0=r_{0}<$ $r_{1}<r_{2}<\cdots$ be the sequence consisting of those $r$ such that $P_{r} \neq P_{r^{+}}$. We put $P^{(n)}=P_{r_{n}}$. Then we have

Lemma (a) $P^{(n)}$ is an F-stable normal subgroup of $P$ (for all $n \geqslant 1$ ) and $P^{(n)} / P^{(n+1)}$ is a finite-dimensional vector space over the residue field $\kappa$.

(b) $P^{(n)}$ is a fundamental system of neighborhoods of the identity element.

(c) for all $i, j \geqslant 1$, the commutator group $\left[P^{(i)}, P^{(j)}\right] \subset\left[P^{(1)}, P^{(j)}\right] \subset$ $P^{(j+1)}$.

(d) $P=\left(P \cap M_{S}\right) \cdot P^{(1)}$ for any $S \in 8$.

(e) The normalizer of $S$ in $P$ is the same as the centralizer in $P$. 
(f) Let $i \geqslant 1, S \in$ 8. Suppose that for each $s \in F$, there exists $u \in P^{(i)}$ such that $s(S)=u^{-1} S u$. Let $Z=M_{S}(k) \cap P^{(i)}$. Then $Z P^{(i+1)}$ is F-stable and $P^{(i)} /\left(Z P^{(i+1)}\right)$ is a pro-p-group.

Proof. (a)-(c) are standard properties of the "Moy-Prasad" filtration, and (d) is due to [MP] as mentioned above. To prove (e), consider an element $g$ in $G(k)$ normalizing $S$. Then $g$ takes $x+V(S) \subset A(H, T)$ to $g . x+V(S) \subset$ $A\left(H, g T g^{-1}\right)$ by an affine transformation whose derivative gives the action of $g$ on $V(S)$. Now if $g \in P$, then $g$ fixes the facet containing $x$ pointwise, which is an open subset of $x+V(S)$. It follows that $g$ acts on $V(S)$ trivially, hence it centralizes $S$.

We now prove (f). Let $s \in F$ and choose $u \in P^{(i)}$ such that $s(S)=$ $u^{-1} S u$. Since $Z$ is the centralizer of $S$ in $P^{(i)}$, we have $s(Z)=u^{-1} Z u$. Therefore we can write any element in $s(Z)$ as $u^{-1} z u$, with $z \in Z$. Since $z\left(z^{-1} u^{-1} z u\right) \in Z\left[P^{(i)}, P^{(i)}\right] \subset Z P^{(i+1)}$, the first statement of (f) is proved.

From [BT1, 6.4.48], there is a decomposition

$$
P^{(i)} / P^{(i+1)}=V_{0} \times \prod_{a \in \Phi} V_{a},
$$

where $\Phi$ is the root system of $(H, T), U_{a}$ the root subgroup of $a \in \Phi$, $V_{a}=\left(U_{a}(k) \cap P^{(i)}\right) /\left(U_{a}(k) \cap P^{(i+1)}\right), V_{0}=\left(Z_{H}(T)(k) \cap P^{(i)}\right) /\left(Z_{H}(T)(k) \cap\right.$ $\left.P^{(i+1)}\right)$. The image of $Z$ in $P^{(i)} / P^{(i+1)}$ is precisely $V_{0} \times \prod_{a \in \Phi^{\prime}} V_{a}$, where $\Phi^{\prime}$ is the root system of $\left(M_{S}, T\right)$. Now the second statement of (f) is clear since each $V_{a}$ has the structure of a finite-dimensional vector space over $\kappa$.

We will now complete the proof of Proposition 1.1.

Choose any $S_{0} \in \delta$. From (d) it follows that every element $S$ of $\delta$ is of the form $u^{-1} S_{0} u$ with $u \in P^{(1)}$. Let $Z_{0}=M_{S_{0}}(k) \cap P^{(1)}$, the normalizer of $S_{0}$ in $P^{(1)}$ (by (e)). Then the coset $Z_{0} u \in Z_{0} \backslash P^{(1)}$ is uniquely determined by $S$.

In particular, for each $s \in F$, we can write $s\left(S_{0}\right)=u_{s}^{-1} S_{0} u_{s}$ with $u_{s} \in P^{(1)}$. It is easy to see that $u_{s t}=u_{s} s\left(u_{t}\right)$ in $Z_{0} \backslash P^{(1)}$. Therefore, $s \mapsto u_{s} \bmod Z_{0} P^{(2)}$ is a 1 -cocycle on $F$ with values in $P^{(1)} / Z_{0} P^{(2)}$. Here, we notice that both $P^{(1)}$ and $Z_{0} P^{(2)}$ are $F$-stable by (a) and (f) of the above lemma. Since $F$ is a finite group of order not divisible by $p$ and $P^{(1)} / Z_{0} P^{(2)}$ is an abelian pro- $p$-group, such a cocycle is a coboundary. This means that we can find $v=v_{0} \in P^{(1)}$ such that $v^{-1} u_{s} s(v) \in Z_{0} P^{(2)}$.

Let $S_{1}=v^{-1} S_{0} v$. Then we have $s\left(S_{1}\right)=u^{-1} S_{1} u$, where $u=v^{-1} u_{s} s(v)$. Let $Z_{1}=Z_{H(k)}\left(S_{1}\right) \cap P^{(1)}$. Then $u \in Z_{0} P^{(2)}=\left(v Z_{1} v^{-1}\right) P^{(2)}=Z_{1} P^{(2)}$ since $P^{(1)} / P^{(2)}$ is abelian. It follows that for each $s \in F$, we can find $u \in P^{(2)}$ such that $s\left(S_{1}\right)=u^{-1} S_{1} u$.

We now repeat the above argument to construct inductively a sequence of tori $\left\{S_{i}\right\}$ in $\delta$, and a sequence of elements $\left\{v_{i}\right\}$ such that 
- For each $s \in F, i \geqslant 0$, there exists $u \in P^{(i+1)}$ such that $s\left(S_{i}\right)=u^{-1} S_{i} u$. $-v_{i} \in P^{(i+1)}$ and $S_{i+1}=v_{i}^{-1} S_{i} v_{i}$ for each $i \geqslant 0$.

Let $w_{i}=v_{0} v_{1} v_{2} \cdots v_{i-1}$ so that $S_{i}=w_{i}^{-1} S_{0} w_{i}$. Since $v_{i} \in P^{(i+1)}$, $w=\lim _{i \rightarrow \infty} w_{i}$ exists. Put $S=w^{-1} S_{0} w$. Fix $s \in F$. For each $i \geqslant 0$, there is an element $u_{i} \in P^{(i+1)}$ such that $s\left(S_{i}\right)=u_{i}^{-1} S_{i} u_{i}$. It follows that $u_{0} s\left(w_{i}\right) u_{i}^{-1} w_{i}^{-1} \in N_{H(k)}\left(S_{0}\right)$. Taking $i \rightarrow \infty$, we have $u_{0} s(w) w^{-1} \in$ $N_{H(k)}\left(S_{0}\right)$. This implies that $s(S)=S$.

Thus $S \in \mathcal{S}$ is $F$-stable and the proposition is proved.

(1.2) Uniqueness of the extended building We recall that the (extended) building $\mathcal{B}(G)$ of $G$ is defined to be $\mathcal{B}\left(G^{\prime}\right) \times E$, where $G^{\prime}$ is the derived group of $G$, and $E=V\left(Z(G)^{\circ}\right)$. Here, we should regard $E$ as an affine space under $V\left(Z(G)^{\circ}\right)$, without a preferred point.

The building is characterized by a few axioms (to be recalled in (1.9.1)) in the following sense: if both $\mathscr{B}^{\prime}$ and $\mathscr{B}^{\prime \prime}$ satisfy the axioms, then there is a bijection $\iota: \mathscr{B}^{\prime} \rightarrow \mathscr{B}^{\prime \prime}$ preserving all the relevant structures; if $\iota_{1}$ and $\iota_{2}$ are such bijections, then $\iota_{1}(x)=\iota_{2}(z \cdot x)$ for some $z \in V\left(Z(G)^{\circ}\right)$. In short, we say that the building is unique up to an isomorphism, which is unique up to translation by an element of $V\left(Z(G)^{\circ}\right)$. The same remark applies to the apartment $A\left(G, S_{0}\right)$ associated to a maximal $k$-split torus $S_{0}$ of $G$.

Thus when we discuss embeddings of buildings $\iota: \mathscr{B}(G) \rightarrow \mathscr{B}(H)$, the best uniqueness statement we can have is that $\iota$ is unique up to translation by an element of $V\left(Z(G)^{\circ}\right)$. This will be the case in (1.3) and (1.9).

(1.3) A variant of the main result Let $S$ be a $k$-split torus of $H$. We say that a (not necessarily finite) subgroup $\mathcal{F}$ of $S(k)_{\mathrm{b}}$ is sufficiently large if $Z_{H}(\mathcal{F})=Z_{H}(S)$ and the following condition holds: for any maximal $k$ split torus $T$ of $H$ containing $S$ and any $x \in A(H, T)$, the centralizer of $\mathcal{F} \bmod \mathfrak{p}$ in $\bar{H}_{x}^{\text {red }}$ is the same as that of $S \bmod \mathfrak{p}$.

It is obvious that any subgroup of finite index of $S(k)_{\mathrm{b}}$ is sufficiently large. Using results of Steinberg [S2], it is easy to see that the $m$-torsion subgroup of $S(k)$ is sufficiently large for $m \gg 0$ prime to $p$.

Now let $\mathcal{F}$ be a sufficiently large subgroup of $S(k)_{\mathrm{b}}$ and $G=Z_{H}(\mathcal{F})=$ $Z_{H}(S)$. Then $G$ is a Levi subgroup of $H$, and it is well-known that there is a natural embedding $j: \mathscr{B}(G) \hookrightarrow \mathscr{B}(H)$ in this case, defined uniquely up to translation by an element of $V\left(Z(G)^{\circ}\right)$.

Proposition The image of the map $j: \mathscr{B}(G) \rightarrow \mathscr{B}(H)$ is $\mathcal{B}(H)^{\mathcal{F}}$.

Proof. It is clear that $j(\mathscr{B}(G)) \subset \mathscr{B}(H)^{\mathcal{F}}$. Assume that the inclusion is proper. Then we can find two chambers $C, C^{\prime}$, both fixed by $\mathcal{F}$, such that their closures share a point $x$ and $C$ lies in $j(\mathscr{B}(G))$ but $C^{\prime}$ does not. There is a maximal $k$-split torus $T$ containing $S$ such that $C$ lies in the apartment of $T$. The group $\bar{H}_{x}^{\text {red }}$ contains a maximal $\kappa$-torus $\bar{T}$ corresponding to $T$ and the chamber $C$ determines a Borel subgroup $\bar{B}$ of $\bar{H}_{x}^{\text {red }}$ containing $\bar{T}$. The 
chamber $C^{\prime}$ also determines a Borel subgroup $\bar{B}^{\prime}$ of $\bar{H}_{x}^{\text {red }}$ containing $\bar{S}$ ( $\bar{S}$ is reduction mod $\mathfrak{p}$ of $S$; it is contained in $\bar{T}$ ). Let $\bar{T}^{\prime}$ be a maximal $\kappa$-torus of $\bar{B}^{\prime}$ containing $\bar{S}$. Then $\bar{T}^{\prime}$ is conjugate to $\bar{T}$ under an element of the centralizer of $\bar{S}$ in $\bar{H}_{x}^{\text {red }}(\kappa)$, which is the image of $G \cap \underline{H}_{x}(\mathcal{O})$ in $\bar{H}_{x}^{\text {red }}(\kappa)$. Hence after replacing $C^{\prime}$ by a conjugate under an element of $G \cap \underline{H}_{x}(\mathcal{O})$, we may assume that $\bar{T}=\bar{T}^{\prime}$. Then $C^{\prime}$ lies in the apartment corresponding to $T$ and hence it is contained in the building of the Levi subgroup $G$, a contradiction.

(1.4) Preliminary remarks In the following, we consider $\mathscr{B}^{\prime}=\mathscr{B}(H)^{F}$. Our goal is to show that this can be identified with $\mathscr{B}(G)$ in a natural way. In particular, we will show that we can see the structure of apartments and facets on $\mathscr{B}^{\prime}$ directly.

The set $\mathscr{B}^{\prime}$ is clearly closed and convex. We now review some notions related to such sets. The dimension of a closed convex subset $E$ in an affine space is defined to be that of the affine subspace spanned by $E$. If $E \subset \mathscr{B}(H)$ is closed and convex, $\operatorname{dim} E$ is defined to be the maximum of $\operatorname{dim} E \cap A(H, T)$ as $T$ ranges over maximal $k$-split tori of $H$.

(1.5) The apartments Let $\mathscr{D}^{\prime}$ be the set of facets $\Delta$ of $\mathscr{B}(H)$ which contain a point fixed under $F$ and let $\mathscr{D}$ be the maximal elements in $\mathscr{D}^{\prime}$ relative to the incidence relation.

By [BT1, 9.2.5], for $\Delta \in \mathscr{D}, \operatorname{dim} \Delta$ and $\operatorname{dim} \Delta^{F}$ are independent of $\Delta$. Thus $\mathscr{D}$ can also be described as the set of elements in $\mathscr{D}^{\prime}$ of maximal dimension.

Let $\Delta \in \mathscr{D}$ and apply Proposition 1.1 to $x \in \Delta^{F}$ to get tori $S \subset T$ satisfying (i) and (ii) of the proposition. Then ([T, 3.5]) $\operatorname{dim} \Delta=\operatorname{dim} Z\left(\bar{H}_{x}^{\mathrm{red}}\right)=$ $\operatorname{dim} S$. Let $M=Z_{H}(S)$, and let $M^{\prime}$ be the derived group of $M$. It follows from (ii) of Proposition 1.1 that $S$ is the maximal $k$-split torus in the center of $M$.

(1.5.1) Lemma (i) The group $F$ fixes a unique point on $\mathscr{B}\left(M^{\prime}\right)$.

(ii) $\operatorname{dim} S^{F}=k$-rank $G$, hence $S_{0}:=\left(S^{F}\right)^{\circ}$ is a maximal $k$-split torus of $G$.

Proof. (i) There is an $F$-equivariant embedding

$$
j: \mathscr{B}\left(M^{\prime}\right) \times V(S)=\mathscr{B}(M) \hookrightarrow \mathscr{B}(H),
$$

where $V(S)=X_{*}(S) \otimes_{\mathbb{Z}} \mathbb{R}$, and the image is $\mathcal{B}(H)^{S(k)_{b}}$. In particular, $x$ is in the image. Let $j^{-1}(x)=(y, v) \in \mathscr{B}\left(M^{\prime}\right) \times V(S)$. Then since $\underline{H}_{x}(\mathcal{O}) \cap M(k)$ is a maximal parahoric subgroup of $M(k)$ [MP, 6.4], $j^{-1}(\Delta)=\{y\} \times E$ for some open subset $E$ of $V(S)$. It follows that $\operatorname{dim} \Delta^{F}=\operatorname{dim} V(S)^{F}=\operatorname{dim} S_{0}$.

Now $\mathscr{B}\left(M^{\prime}\right)^{F}$ is a non-empty closed convex subset of $\mathcal{B}\left(M^{\prime}\right)$. If it contains more than one point, then its dimension is positive. Consider a point $y^{\prime} \in \mathcal{B}\left(M^{\prime}\right)^{F}$ close to $y$. The point $j\left(y^{\prime}, v\right)$ is fixed by $F$ and does not lie on the closure $\bar{\Delta}$ of $\Delta$ if $y \neq y^{\prime}$. Thus the facet $\Delta^{\prime}$ of $\mathcal{B}(H)$ containing $j\left(y^{\prime}, v\right)$ 
does not lie on $\bar{\Delta}$. For $y^{\prime}$ sufficiently close to $y, \Delta^{\prime}$ has to be a facet whose closure contains $\Delta$, contradicting our choice of $\Delta$.

(ii) Let $S_{1}$ be a maximal $k$-split torus of $G$ containing $S_{0}$ and let $M_{1}=$ $Z_{H}\left(S_{1}\right)$. Again there is an $F$-equivariant embedding

$$
j_{1}: \mathscr{B}\left(M_{1}\right) \rightarrow \mathscr{B}(H) .
$$

It is clear that for any $y \in \mathscr{B}\left(M_{1}\right)^{F}$, the convex hull of $S_{1}(k) . y$ is an affine space of dimension $=\operatorname{dim} S_{1}$. This forces $\operatorname{dim} \mathcal{B}(H)^{F} \geqslant \operatorname{dim} S_{1}$. On the other hand, it is also clear (from [BT1, 9.2.5]) that $\operatorname{dim} \mathscr{B}(H)^{F}=\operatorname{dim} \Delta^{F}=$ $\operatorname{dim} S_{0}$. Thus we must have $\operatorname{dim} S_{0}=\operatorname{dim} S_{1}$ and $S_{0}=S_{1}$.

(1.5.2) Corollary With the above notations, put $M_{0}=Z_{H}\left(S_{0}\right)$. The inclusions $\mathcal{B}(M) \subset \mathscr{B}\left(M_{0}\right) \subset \mathcal{B}(H)$ induced from $M \subset M_{0} \subset H$ give rise to the following relation

$$
\mathscr{B}(M)^{F}=\mathscr{B}\left(M_{0}\right)^{F} \subset \mathscr{B}(H)^{F},
$$

moreover, $\mathcal{B}(M)^{F}=\mathscr{B}\left(M_{0}\right)^{F}$ is an affine space of dimension $=k$-rank $G$.

Proof. By part (i) of the lemma, it is clear that $\mathscr{B}(M)^{F}=\mathscr{B}\left(M^{\prime}\right)^{F} \times$ $V(S)^{F}=y \times V\left(S_{0}\right)$ is an affine space of dimension $=\operatorname{dim} S_{0}$. On the other hand, this dimension is also equal to $\operatorname{dim} \Delta^{F}=\operatorname{dim} \mathscr{B}(H)^{F}$.

Let $S_{0}^{\prime}$ be the maximal $k$-split torus in the center of $M_{0}$. Clearly $S_{0}^{\prime} \supset$ $S_{0}$. We have $\mathcal{B}\left(M_{0}\right)^{F}=\mathscr{B}\left(M_{0}^{\prime}\right)^{F} \times V\left(S_{0}^{\prime}\right)^{F} \supset \mathscr{B}\left(M_{0}^{\prime}\right)^{F} \times V\left(S_{0}\right)$. Since $\mathscr{B}\left(M_{0}\right)^{F} \subset \mathscr{B}(H)^{F}$, it has dimension at most $\operatorname{dim} S_{0}$. Thus the convex set $\mathscr{B}\left(M_{0}^{\prime}\right)^{F}$ is 0 -dimensional, and must reduce to a single point.

(1.5.3) Now we see that $\mathcal{B}\left(M_{0}\right)^{F}=\mathscr{B}(M)^{F} \subset \mathscr{B}^{\prime}$ is an affine space of dimension $=k$-rank $G$. It should certainly be the apartment $A\left(G, S_{0}\right)$ in the identification of $\mathscr{B}(G)$ with $\mathscr{B}^{\prime}$ we wish to establish. Therefore, we will denote it by $A^{\prime}\left(G, S_{0}\right)$.

(1.5.4) Remark When $F$ is cyclic, the following fact is quite remarkable: Every $\Delta$ in $D$ is a chamber. Consequently, $S=T$ is a maximal $k$-split torus of $H$, and every maximal $k$-split torus $S_{0}$ of $G$ is contained in an F-stable maximal $k$-split torus $T$.

To see this, we start with any $z \in \mathscr{B}(H)^{F}$. Then $F$ acts on $\underline{H}_{z}$ and $\bar{H}_{z}^{\text {red }}$. By a theorem of Steinberg [S1], there exists an $F$-stable Borel subgroup $B$ of $\bar{H}_{z}^{\text {red }}$. The inverse image of $B(\kappa)$ in $\underline{H}_{z}(\mathcal{O})$ is an $F$-stable Iwahori subgroup, whose corresponding chamber $C$ is $F$-stable and contains $z$ in its closure. Obviously, the center of mass of $C$ is fixed by $F$ and hence $C^{F}$ is non-empty.

(1.6) The action of the affine Weyl group Let $A=A\left(G, S_{0}\right), Z=$ $Z_{G}\left(S_{0}\right)$, and $N=N_{G}\left(S_{0}\right)$. We recall that $A$ is an affine space under $V_{0}=$ $V\left(S_{0}\right)$, and $N(k)$ acts on $A$ by affine transformations. Denote by $\operatorname{Aff}(A)$ the 
group of affine automorphisms of $A$ and $v: N(k) \rightarrow \operatorname{Aff}(A)$ the action map, then

A1 The maximal bounded subgroup $Z(k)_{\mathrm{b}}$ of $Z(k)$ acts trivially: $v\left(Z(k)_{\mathrm{b}}\right)$ $=\{1\}$.

A2 The group $Z(k)$ acts by translations, and the action is characterized by the following formula: for $s \in S_{0}(k)$,

$$
\chi(v(s))=- \text { ord } \chi(s) \quad \text { for all } \chi \in X^{*}(S),
$$

here we regard the translation $v(s)$ as an element of $V_{0}=X_{*}\left(S_{0}\right) \otimes_{\mathbb{Z}} \mathbb{R}$. A3 For $g \in \operatorname{Aff}(A)$, denote by $d g \in \operatorname{GL}\left(V_{0}\right)$ the derivative of $g$. Then the map $d v: N \rightarrow \mathrm{GL}\left(V_{0}\right)$ is induced from the action of $N$ on $X_{*}\left(S_{0}\right)$ (i.e. the Weyl group action).

Moreover, these three conditions characterize the affine space, up to translation by an element of $V\left(Z(G)^{\circ}\right)$. See [T, 1.2].

We now consider $A^{\prime}=A^{\prime}\left(G, S_{0}\right)$ as defined in (1.5.3), which is also an affine space under $V_{0}$. Since $A^{\prime}=\mathscr{B}\left(M_{0}\right)^{F}$ and $M_{0}=Z_{H}\left(S_{0}\right)$, it is obviously $N(k)$-invariant. We will show that the action is affine and satisfies $\mathbf{A 1}, \mathbf{A 2}$, and A3.

Lemma The group $N(k)$ acts on $A^{\prime}$ by affine transformations. Denote the action map by $v^{\prime}: N(k) \rightarrow \operatorname{Aff}\left(A^{\prime}\right)$. Then the conditions $\mathbf{A 1}, \mathbf{A 2}$, and $\mathbf{A 3}$ are satisfied with $(v, A)$ replaced by $\left(v^{\prime}, A^{\prime}\right)$.

Proof. Let $T$ be any maximal $k$-split torus of $H$ containing $S_{0}$ such that $A(H, T)$ contains $A^{\prime}$. Then the action of $g \in N(k)$ on $\mathcal{B}(H)$ takes $A(H, T)$ to $A\left(H, g T^{-1}\right)$ via an affine isomorphism $\tilde{v}(g)$. Therefore, the action takes the affine subspace $A^{\prime} \subset A(H, T)$ to $A^{\prime} \subset A\left(H, g T g^{-1}\right)$ by an affine isomorphism as well.

The derivative $d \tilde{v}(g): V(T) \rightarrow V\left(g T g^{-1}\right)$ is induced from the map $X_{*}(T) \rightarrow X_{*}\left(g T g^{-1}\right), \lambda \mapsto$ Int $g \circ \lambda$, where Int $g$ is the inner automorphism determined by $g$. Therefore, the restriction $d \nu^{\prime}(g): V_{0} \rightarrow V_{0}$ is induced from $X_{*}\left(S_{0}\right) \rightarrow X_{*}\left(S_{0}\right), \lambda \mapsto$ Int $g \circ \lambda$. This proves $\mathbf{A 3}$.

The condition $\mathbf{A 3}$ implies that $d v^{\prime}$ is trivial on $Z(k)$. Therefore, $Z(k)$ acts by translations. The action of the bounded subgroup $Z(k)_{\mathrm{b}}$ on $A^{\prime}$ admits a fixed point by the fixed point theorem [BT1, 3.2.4]. Therefore, $Z(k)_{\mathrm{b}}$ acts by the trivial translation. This proves A1.

Finally, if $g=s$ is in $S_{0}(k)$, then $g T g^{-1}=T$ and the translation $\tilde{v}(g)$ satisfies

$$
\chi(\tilde{v}(g))=-\operatorname{ord} \chi(g) \quad \text { for all } \chi \in X^{*}(T) .
$$

Since $\tilde{v}(g)=v^{\prime}(s)$ and $X^{*}(T) \rightarrow X^{*}\left(S_{0}\right)$ is surjective, condition $\mathbf{A 2}$ is now clear. 
By the characterization of the apartment $A\left(G, S_{0}\right)$, there is an isomorphism $A \rightarrow A^{\prime}$ of affine spaces with $N(k)$-actions, unique up to translation by an element of $V\left(Z(G)^{\circ}\right)$.

\section{(1.7) The fixed points of parahoric subgroups}

Proposition Let $y \in \mathscr{B}(G)$ and $P=\underline{G}_{y}(\mathcal{O})$ the corresponding parahoric subgroup. Then the convex subset $\left(\mathscr{B}^{\prime}\right)^{P}$ of $\mathscr{B}^{\prime}$ fixed by $P$ is of dimension $\leqslant \operatorname{dim} Z\left(\bar{G}_{y}^{\text {red }}\right)$.

We begin with a lemma, which should be well-known. The lemma concerns an arbitrary connected reductive group $G$ over $k$, and it is valid over any discretely valuated complete field $k$.

Lemma Let $P$ be a parahoric subgroup of $G(k)$ and $K$ be a bounded subgroup of $G(k)$ containing $P$. Then there is a parahoric subgroup $P^{\prime}$ of $G(k)$ such that $P \subset P^{\prime} \subset K$ and $\left[K: P^{\prime}\right]<\infty$.

Proof. Consider $\mathcal{B}(G)^{K}$. This is a subset of the closure of the facet corresponding to $P$. Choose a point $z$ in this set such that the facet $F$ containing $z$ is of maximal dimension. Let $P^{\prime}$ be the corresponding parahoric subgroup, and $P^{\prime \prime}$ the subgroup of $G(k)$ fixing $z$. So we have $P \subset P^{\prime}, K \subset P^{\prime \prime}$, $\left[P^{\prime \prime}: P^{\prime}\right]<\infty$, and $P^{\prime}$ is normal in $P^{\prime \prime}$. It remains to show that $P^{\prime} \subset K$.

Consider $K^{\prime}=K \cap P^{\prime}$. Then $\left[K: K^{\prime}\right]$ is finite. Clearly $K^{\prime} \supset P^{+} \supset$ $\left(P^{\prime}\right)^{+}$. The image of $K^{\prime}$ in $P^{\prime} /\left(P^{\prime}\right)^{+}$is a parabolic subgroup as it contains the image of $P$, which is parabolic. Thus $K^{\prime}$ is also a parahoric subgroup. If $K^{\prime}$ is not $P^{\prime}$, its corresponding facet $F^{\prime}$ is of dimension $>\operatorname{dim} F$.

Since $K^{\prime}$ is normal in $K, K$ permutes the set of points fixed by $K^{\prime}$, which is just the closure of $F^{\prime}$. Now it is clear that $K$ fixes the center of mass of $F^{\prime}$, contradicting our choice of $z$.

Thus $K^{\prime}=P^{\prime}$ and hence $P^{\prime} \subset K$.

Proof of Proposition 1.7. Let us choose a facet $\Delta$ in $\mathscr{B}(H)$ which contains a point of $\left(\mathscr{B}^{\prime}\right)^{P}$ but no bigger facet does. Then $\Delta$ is $F$-stable. Let $x \in$ $\Delta \cap\left(\mathscr{B}^{\prime}\right)^{P}$ and let $Q=\underline{H}_{x}(\mathcal{O})$. Since $P$ fixes $x, P$ is contained in $Q^{\prime}$, the subgroup of $H(k)$ fixing $x$. By Bruhat-Tits, there is a smooth group scheme $\underline{H}_{x}^{\prime}$ such that $\underline{H}_{x}^{\prime}(\mathcal{O})=Q^{\prime}$ and $\left(\underline{H}_{x}^{\prime}\right)^{\circ}=\underline{H}_{x}$. The inclusion $P \hookrightarrow Q^{\prime}$ induces a morphism $\underline{G}_{y} \rightarrow \underline{H}_{x}^{\prime}$, which factor through $\underline{H}_{x}$ since $\underline{G}_{y}$ is connected. Thus we have $P \subset Q$. This shows that $P$ fixes $\Delta$ pointwise. By [BT1, 9.2.5], $\operatorname{dim}\left(\mathscr{B}^{\prime}\right)^{P}=\operatorname{dim} \Delta \cap\left(\mathcal{B}^{\prime}\right)^{P}=\operatorname{dim} \Delta^{F}$.

We now apply Proposition 1.1 to $x$ to obtain tori $S \subset T$ satisfying (i) and (ii) of that proposition. As we have mentioned in the proof of Proposition 1.1, $Z_{H}(S)(k) \cap Q$ is a maximal parahoric subgroup of $Z_{H}(S)(k)$. It follows that $Z_{H}(S)(k) \cap Q$ fixes essentially one point in $\mathscr{B}\left(Z_{H}(S)\right)$. More precisely, if $x_{0}$ is one such fixed point, the set of all fixed points is then $V \cdot x_{0}$, where $V=X_{*}(S) \otimes_{\mathbb{Z}} \mathbb{R}$. We may and do assume that $x_{0}$ is fixed by $F$. 
By construction, $\Delta \subset V \cdot x_{0}$ and hence

$$
\operatorname{dim}\left(\mathcal{B}^{\prime}\right)^{P}=\operatorname{dim} \Delta^{F} \leqslant \operatorname{dim} V^{F}=\operatorname{dim} V_{0}=\operatorname{dim} S_{0},
$$

where $V_{0}=V^{F}=X_{*}\left(S_{0}\right) \otimes_{\mathbb{Z}} \mathbb{R}$, and $S_{0}$ is $\left(S^{F}\right)^{\circ}$. Now it suffices to show that $\operatorname{dim} S_{0} \leqslant \operatorname{dim} Z\left(\bar{G}_{y}^{\text {red }}\right)$.

It follows from the preceding lemma that there is a parahoric subgroup $P^{\prime}=G_{z}(\mathcal{O})$ such that $P \subset P^{\prime} \subset Q \cap G(k)$ and $P^{\prime}$ is of finite index in $Q \cap G(k)$. Now $P^{\prime} \cap S_{0}(k)_{\mathrm{b}}$ is a subgroup of finite index in $S_{0}(k)_{\mathrm{b}}$, and as such, it is sufficiently large in $S_{0}(k)_{\mathrm{b}} \subset G(k)$ in the sense of (1.3). Thus we have $z \in A\left(G, S_{0}\right)$ and hence actually $S_{0}(k)_{\mathrm{b}} \subset P^{\prime}$. Since $\operatorname{dim} Z\left(\bar{G}_{z}^{\text {red }}\right) \leqslant$ $\operatorname{dim} Z\left(\bar{G}_{y}^{\mathrm{red}}\right)$, it suffices to show that $\operatorname{dim} S_{0} \leqslant \operatorname{dim} Z\left(\bar{G}_{z}^{\text {red }}\right)$.

The morphism $\underline{G}_{z} \rightarrow \underline{H}_{x}$ (induced by $P^{\prime} \hookrightarrow Q$ ) induces a map $\underline{G}_{z}(\mathcal{O}) \rightarrow \bar{H}_{x}^{\text {red }}(\kappa)$, with kernel $K=P^{\prime} \cap Q^{+}$. Since $Q^{+}$is a pro- $p$ group, so is $K$. Thus we have an injection $P^{\prime} / K \hookrightarrow \bar{H}_{x}^{\text {red }}(\kappa)$ and $\bar{G}_{z}^{\text {red }}(\kappa)$ is a quotient of $P^{\prime} / K$.

By construction, $S$ reduces to a torus $\bar{S}$ in $\bar{H}_{x}^{\text {red }}$ of the same dimension, and is the central torus of the latter. Similarly, $S_{0}$ reduces to a torus $\bar{S}_{0}$ of $\bar{G}_{z}^{\text {red }}$. Observe that $S_{0}(k)_{\mathrm{b}} \cap K$ is precisely the kernel of the reduction map $S_{0}(k)_{\mathrm{b}} \rightarrow \bar{S}(\kappa)$. Now the commutativity of the diagram

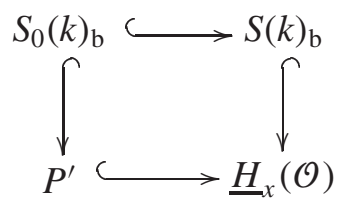

implies that of

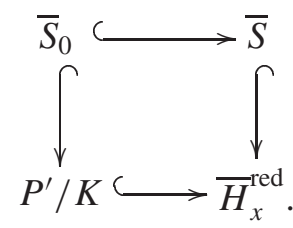

Thus we have $\bar{S}_{0} \subset Z\left(\bar{G}_{z}^{\text {red }}\right)$ and $\operatorname{dim} S_{0}=\operatorname{dim} \bar{S}_{0} \leqslant Z\left(\bar{G}_{z}^{\text {red }}\right)$.

(1.8) A compatibility We maintain the notations of (1.6). Let $y$ be a vertex lying on $A=A\left(G, S_{0}\right)$ and $P=\underline{G}_{y}(\mathcal{O})$. It is clear that the set $\left(\mathscr{B}^{\prime}\right)^{P}$ is $Z(G)(k)$-invariant, and $V\left(Z(G)^{\circ}\right)$ acts on it by translations. Thus this set is of dimension $\geqslant \operatorname{dim} V\left(Z(G)^{\circ}\right)=\operatorname{dim} Z\left(\bar{G}_{y}^{\text {red }}\right)$. Thus we conclude, by (1.7), that $P$ fixes a unique point $y^{\prime}$ on $\mathscr{B}^{\prime}$, modulo the action of $V\left(Z(G)^{\circ}\right)$. Since $P \supset S_{0}(k)_{\mathrm{b}}$, the point $y^{\prime}$ lies on $\left(\mathscr{B}(H)^{S_{0}(k)_{\mathrm{b}}}\right)^{F}=A^{\prime}$ by $(1.3)$.

This gives us a second way of identifying $A$ with $A^{\prime}$, namely by sending $y$ to $y^{\prime}$. We would like to show that this is the same as the identification 
constructed in (1.6). In particular, it doesn't depend on the choice of the vertex on $A$.

Choose a chamber $C$ on $A$ whose closure contains $y$, and let $I$ be the corresponding Iwahori subgroup. Then $I$ determines a system of simple affine roots, and we can choose elements $n_{0}, \ldots, n_{r}$ in $N(k)$ representing the affine reflections corresponding to these simple affine roots. We may and do assume that $P$ is generated by $I$ and $n_{1}, \ldots, n_{r}$.

Since $y$ is fixed by $P$, it is fixed by $n_{1}, \ldots, n_{r}$. And in fact, it can be characterized as the unique point (modulo $V\left(Z(G)^{\circ}\right)$ ) on $A$ fixed by $n_{1}, \ldots, n_{r}$. Similarly, $y^{\prime}$ is the unique point (modulo $V\left(Z(G)^{\circ}\right)$ ) on $A^{\prime}$ fixed by $n_{1}, \ldots, n_{r}$. This shows the desired compatibility.

(1.9) The main theorem We now drop the assumption that $k$ is strictly henselian and state our main theorem.

Theorem Assume that \#F is not divisible by the residue characteristic of $k$. There is a $G(k)$-equivariant toral map $\iota: \mathscr{B}(G) \rightarrow \mathscr{B}(H)$ whose image is $\mathscr{B}(H)^{F}$, uniquely defined up to translation by an element of $V\left(Z(G)^{0}\right)$, and compatible with unramified change of base field.

Here, we are using the notion of a toral map $\iota$ defined by Landvogt. It means that for every maximal $k$-split torus $S_{0}$ of $G$, there is a maximal $k$-split torus $T$ of $H$ such that $S_{0} \subset T$ and $\iota$ maps $A\left(G, S_{0}\right)$ to $A(H, T)$ by an affine transformation.

We first give two proofs assuming that $k$ is strictly henselian.

(1.9.1) The first proof Our first approach is based on the following characterization of $\mathscr{B}(G)$ given in [T, 2.1]:

The building $\mathscr{B}(G)$ is the unique $G(k)$-set which contains a subset A satisfying:

B1 As an $N(k)$-set, $A$ is isomorphic to $A\left(G, S_{0}\right)$, characterized by $\mathbf{A 1}, \mathbf{A 2}$, and $\mathbf{A 3}$ of (1.6).

B2 $\mathscr{B}(G)=\bigcup_{g \in G(k)} g . A$.

B3 For each affine root $\alpha$ of $\left(G, S_{0}\right)$, the affine root subgroup $X_{\alpha}$ (described in $[T, 1.4])$ fixes the half-apartment $\alpha^{-1}([0, \infty)) \subset$ $A \simeq A\left(G, S_{0}\right)$ pointwise.

Here the uniqueness is as explained in (1.2).

We claim that $\mathscr{B}^{\prime}=\mathscr{B}(H)^{F}$ satisfies these conditions with $A^{\prime}=$ $A^{\prime}\left(G, S_{0}\right)$ playing the role of $A$. Indeed, B1 is established in (1.6), and B2 is clear from (1.5).

Let us verify B3. It suffices to show that $X_{\alpha}$ fixes every vertex $y$ in $\alpha^{-1}([0, \infty))$. The condition $y \in \alpha^{-1}([0, \infty))$ is equivalent to $X_{\alpha} \subset \underline{G}_{y}(\mathcal{O})$. By the discussion in (1.8), $\underline{G}_{y}(\mathcal{O})$ does fix the point $y^{\prime}$ on $A^{\prime}$ corresponding to $y$ via the identification of $A^{\prime}$ with $A\left(G, S_{0}\right)$.

Thus we have shown that $\mathcal{B}(H)^{F}$ can be identified with $\mathcal{B}(G)$ canonically. It is clear that the identification map is $G(k)$-equivariant and toral. 
(1.9.2) The second proof We now give another argument. The idea is that we already know how to map the apartments, modulo $V\left(Z(G)^{\circ}\right)$. It suffices to make sure that we can glue them consistently. First we would like to deal away the fact that the identification of $A\left(G, S_{0}\right)$ with $A^{\prime}\left(G, S_{0}\right)$ is only unique modulo $V\left(Z(G)^{\circ}\right)$.

Let $Z$ be the maximal $k$-split torus in $Z(G)$ and let $M=Z_{H}(Z)$. We claim that $\mathcal{B}(H)^{F} \subset \mathscr{B}(M)$. Indeed, we know that every $x \in \mathscr{B}(H)^{F}$ is contained in $A^{\prime}\left(G, S_{0}\right)$ for some maximal $k$-split torus $S_{0}$ of $G$. In particular, $x$ is fixed by $S_{0}(k)_{\mathrm{b}} \supset Z(k)_{\mathrm{b}}$. This implies that $x \in \mathscr{B}(M)$ by Proposition (1.3).

Thus we can replace $H$ by $M$. Now $Z \subset Z(H)$ and $V(Z)$ acts on both $\mathscr{B}(G)$ and $\mathscr{B}(H)$ by translations, and it suffices to construct a unique map $\bar{\imath}: \mathscr{B}(G) / V(Z) \rightarrow \mathscr{B}(H)^{F} / V(Z)$. Now (1.6) gives us a unique identification $\bar{\iota}_{S_{0}}$ of $A\left(G, S_{0}\right) / V(Z)$ with $A^{\prime}\left(G, S_{0}\right) / V(Z)$ for every maximal $k$-split torus $S_{0}$ of $G$. We claim that the $\bar{\iota}_{S_{0}}$ 's glue together to give a map $\bar{\imath}: \mathscr{B}(G) / V(Z) \rightarrow \mathscr{B}(H)^{F} / V(Z)$.

By [BT1, 7.4.8], to show that $\bar{\iota}_{S_{0}}=\bar{\iota}_{S_{1}}$ on $A\left(G, S_{0}\right) \cap A\left(G, S_{1}\right)$ for two maximal $k$-split tori $S_{0}$ and $S_{1}$, it suffices to observe that $\bar{\iota}_{S_{0}}(y)=\bar{\iota}_{S_{1}}(y)$ for all vertices $y \in A\left(G, S_{0}\right) \cap A\left(G, S_{1}\right)$. By (1.8), we know that $\bar{\iota}_{S_{0}}(y)$ and $\bar{\iota}_{S_{1}}(y)$ can both be described as the unique point in $\mathcal{B}(H)^{F} / V(Z)$ fixed by $\underline{G}_{y}(\mathcal{O})$. This proves the claim.

Thus we have obtained a map $\iota: \mathscr{B}(G) \rightarrow \mathscr{B}(H)^{F}$, which is injective (looking at an apartment containing two given points) and surjective (by (1.5)), $G(k)$-equivariant, and toral (also clear from (1.5)).

(1.9.3) The general case We now treat the case of a general $k$. Let $K / k$ be the maximal unramified extension. Then the theorem has been established over $\hat{K}$, the completion of $K$. It is well-known [L, 2.1.3] that $\mathscr{B}(G \otimes K)$ $=\mathscr{B}(G \otimes \hat{K})$. Therefore, the theorem is also true over $K$. Let $X_{K}$ be the set of $G(K)$-equivariant toral maps $\iota_{K}: \mathscr{B}(G \otimes K) \rightarrow \mathscr{B}(H \otimes K)$ constructed by the theorem. We know that $X_{K}$ is an affine space under $V\left(Z(G \otimes K)^{\circ}\right)$. It follows that we can find $\iota_{K} \in X_{K}$ which is $\Gamma$-equivariant, where $\Gamma=\operatorname{Gal}(K / k)$.

Lemma Let $G \subset H$ be connected reductive groups over $k$ and let $\iota_{K}$ be a $G(K) \rtimes \Gamma$-equivariant toral map from $\mathscr{B}(G \otimes K)$ into $\mathscr{B}(H \otimes K)$. Let $\iota$ be the restriction of $\iota_{K}$ on $\Gamma$-fixed points, from $\mathscr{B}(G \otimes K)^{\Gamma}=\mathscr{B}(G)$ to $\mathscr{B}(H \otimes K)^{\Gamma}=\mathcal{B}(H)$. Then เ is toral.

Proof. This follows from [BT2, 5.1.10], or the following simple argument. Let $S_{0}$ be a maximal $k$-split torus of $G$, then $\mathcal{B}(H \otimes K)^{S_{0}(K)_{\mathrm{b}}}$ is precisely $\mathscr{B}\left(M_{0} \otimes K\right)$ by Proposition (1.3), where $M_{0}=Z_{H}\left(S_{0}\right)$. In particular, $\iota\left(A\left(G, S_{0}\right)\right) \subset \mathscr{B}\left(M_{0} \otimes K\right)^{\Gamma}=\mathscr{B}\left(M_{0}\right)$. Pick $x \in A\left(G, S_{0}\right)$ and let $T \supset S_{0}$ be a maximal $k$-split torus of $M_{0}$ such that $\iota(x) \in A\left(M_{0}, T\right)$. Then $\iota\left(A\left(G, S_{0}\right)\right)$, the convex hull of $S_{0}(k) . \iota(x)$, is contained in $A\left(M_{0}, T\right)=$ $A(H, T)$ (here, we observe that, being toral, $\iota_{K}$ (hence also $\iota$ ) respects the formation of convex hulls). This shows that $\iota$ is toral. 
The above lemma gives the desired toral map $\iota: \mathscr{B}(G) \rightarrow \mathscr{B}(H)^{F}$. Moreover, it is also clear that the set of all such $\iota$ 's is an affine space under $\left(X_{*}\left(Z(G \otimes K)^{\circ}\right) \otimes \mathbb{R}\right)^{\Gamma}=X_{*}\left(Z(G)^{\circ}\right) \otimes \mathbb{R}$. The theorem is proved.

(1.10) Remark It is clear from (1.7) that the natural $G(k)$-equivariant toral maps $\iota$ given by the theorem are characterized by the property that $\iota$ extends to a $G(K)$-equivariant toral map $\mathscr{B}(G \otimes K) \rightarrow \mathscr{B}(H \otimes K)$ whose image lies in $\mathcal{B}(H \otimes K)^{F}$.

(1.11) Remark The building $\mathscr{B}(G)$ has a canonical rational structure: there is a subset $\mathscr{B}(G)_{\mathbb{Q}}$ of rational points on $\mathscr{B}(G)$, which is the union of apartments $A\left(G, S_{0}\right)_{\mathbb{Q}}$, and the latter is an affine space under $V\left(S_{0}\right)_{\mathbb{Q}}=$ $X_{*}\left(S_{0}\right) \otimes_{\mathbb{Z}} \mathbb{Q}$.

From our argument, it is clear that the isomorphism $\iota: \mathscr{B}(G) \rightarrow \mathscr{B}(H)^{F}$ can be made to respect the rational structure: $\iota\left(\mathscr{B}(G)_{\mathbb{Q}}\right)=\left(\mathscr{B}(H)_{\mathbb{Q}}\right)^{F}$, and the set of such $\iota$ 's is a principal homogeneous space of $X_{*}\left(Z(G)^{\circ}\right) \otimes_{\mathbb{Z}} \mathbb{Q}$.

\section{Reductivity of $G$}

In this section, $k$ can be an arbitrary field.

(2.1) Theorem Assume that $\# F$ is not divisible by the characteristic of $k$. Then $G=\left(H^{F}\right)^{\circ}$ is a reductive group.

We will give two proofs of this theorem. The characteristic 0 case is easy by reducing to $k=\mathbb{C}$ and making use of the symmetric space. Our first proof of the characteristic $p$ case has a similar flavor in that it makes use of the Bruhat-Tits building and our main Theorem (1.9) in an interesting way. Thus we include it here even though our second proof is short and characteristic-free.

(2.2) The characteristic 0 case Suppose that $k$ is of characteristic 0 . Without loss of generalities, we may assume that $k=\mathbb{C}$. Let $\&$ be the set of compact real forms of $H$. Then $\delta$ is a Riemannian symmetric space. The group $F$ acts on $\&$ by transport of structures.

By the fixed-point theorem, $F$ fixes a point on $\&$. This means that the corresponding compact real form $\underline{H}$ of $H$ is $F$-stable. Since all automorphism of the compact group $\underline{H}(\mathbb{R})$ are defined over $\mathbb{R}, F$ is now a subgroup of $\operatorname{Aut}_{\mathbb{R}}(\underline{H})$. Clearly, $\underline{H}^{F}$ is a real form of $H^{F}$, and $\underline{H}^{F}(\mathbb{R})$ is compact. Therefore, $H^{F}$ is reductive.

(2.3) The characteristic $p$ case Suppose that $k$ is of characteristic $p>0$ and algebraically closed. The automorphism group $J=\operatorname{Aut}(H)$ is an extension of a discrete group by the adjoint group $H^{\text {ad }}$ of $H$.

All these groups admit Chevalley models. In particular, we have group schemes $\underline{H}, \underline{J}, \underline{H}^{\text {ad }}$ over $\mathcal{O}_{0}:=W(k)$, the ring of Witt vectors over $k$. The 
group scheme $\underline{J}$ is an extension of a (discrete) constant group scheme by $\underline{H}^{\text {ad }}$, and acts on $\underline{H}$ by automorphisms.

The reduction map $\underline{J}\left(\mathcal{O}_{0}\right) \rightarrow J(k)$ is surjective and the kernel is a pro$p$-group. Since $(p, \# F)=1$, it follows that the subgroup $F$ of $J(k)$ lifts to a subgroup of $\underline{J}\left(\mathcal{O}_{0}\right)$, which we still denote by $F$.

Let $k_{0}=\operatorname{Frac} \mathcal{O}_{0}, H_{0}=\underline{H} \otimes_{\mathcal{O}_{0}} k_{0}$, and let $G_{0}=\left(H_{0}^{F}\right)^{\circ}$. Since $k_{0}$ is of characteristic $0, G_{0}$ is reductive by (2.2). The subgroup $\underline{H}\left(\mathcal{O}_{0}\right)$ determines a hyperspecial point $y$ on $\mathcal{B}\left(H_{0}\right)$, which is fixed by $F$. By our main theorem ((1.9), which is applicable since $G_{0}$ is known to be reductive), $y=\iota(x)$ for some $x \in \mathscr{B}\left(G_{0}\right)$, where $\iota: \mathscr{B}\left(G_{0}\right) \rightarrow \mathscr{B}\left(H_{0}\right)$ is the canonical embedding constructed by the main theorem.

By (1.11), for a sufficiently ramified extension $k_{1}$ of $k_{0}$, the point $x$ becomes a hyperspecial point on $\mathscr{B}\left(G_{0} \otimes k_{1}\right)$. By a theorem of Edixhoven [E], $\underline{G_{0} \otimes k_{1}} x$ is the identity component of $\left(\underline{H}_{0} \otimes k_{1} y\right)^{F}$. But the special fiber of the latter is just $H^{F}$, and the special fiber of the former is a reductive group $G$, and we have $G=\left(H^{F}\right)^{\circ}$. This proves that $\left(H^{F}\right)^{\circ}$ is reductive.

(2.4) A characteristic-free proof Again we assume that $k$ is algebraically closed. Let $H_{1}=H \rtimes F$ and consider the set $R\left(F, H_{1}\right)$ of homomorphisms of $F$ into $H_{1}$. This set has an obvious structure of an affine algebraic variety. The group $H$ (resp. $\left.H_{1}\right)$ acts on $R\left(F, H_{1}\right)$ by conjugation, and the stabilizer of the canonical homomorphism $e: F \rightarrow H \rtimes F, s \mapsto 1 \rtimes s$ is precisely $H^{F}$ (resp. an extension of a finite group by $H^{F}$, call it $G_{1}$ ).

By [PR, Theorem 2.17], there are finitely many $H_{1}$-orbits in $R\left(F, H_{1}\right)$, and they are all closed (it is assumed in [PR] that $k$ is of characteristic 0 , but the weaker assumption $p \nmid \# F$ suffices by examining the proof there). In particular, the orbit of $e$ is closed, hence affine. It follows (see, e.g. [R]) that $G_{1}$, hence also $G=\left(G_{1}\right)^{\circ}$, is reductive.

(2.5) Remark Assume that $k$ is of characteristic $p>0$. Then if $p$ divides the order of $F$, it is not true in general that $G=\left(H^{F}\right)^{\circ}$ is a reductive group. For example, if $H=\mathrm{SL}_{2}$ and $F$ is a subgroup of the group of upper triangular unipotent matrices of $\mathrm{SL}_{2}(k)$ of order $p$, then the identity component of the centralizer of $F$ in $H$ is the group of upper triangular unipotent matrices.

\section{Spherical buildings}

(3.1) Setting We maintain all the basic notation and assumptions about $k, G, H, F$, except that now $k$ is assumed to be an arbitrary field, and $p$ is the characteristic of $k$.

(3.2) Spherical buildings Let $\delta(G)$ be the spherical building of $G$ as defined in [CLT]. We now briefly recall the construction of $\delta(G)$ as a set. 
For each maximal $k$-split torus $S$ of $G$, let $s(S)=\left(X_{*}(S) \otimes\right.$ $\mathbb{R} \backslash\{0\}) / \mathbb{R}_{>0}$, where $\mathbb{R}_{>0}$ acts on $X_{*}(S) \otimes \mathbb{R} \backslash\{0\}$ by scalar multiplications. It is well-known that for each $b \in \delta(S)$, one can associate a parabolic subgroup $P(b)$ of $G$ (see [CLT, §1], also the proof of Lemma 3.3 below). We define an equivalence relation $\sim$ on the disjoint union $\bigsqcup_{S} \delta(S)$ (taken over all maximal $k$-split tori of $G$ ) as follows: for $b \in \delta(S), b^{\prime} \in \delta\left(S^{\prime}\right), b \sim b^{\prime}$ if and only if there exists $g \in P(b)(k)$ such that $S^{\prime}=g S g^{-1}$ and $b^{\prime}=g \cdot b$. Here, we notice that $\lambda \mapsto \operatorname{Int} g \circ \lambda$ maps $X_{*}(S)$ to $X_{*}\left(g S g^{-1}\right)$, and we have denoted the extension $X_{*}(S) \otimes \mathbb{R} \rightarrow X_{*}\left(g S g^{-1}\right) \otimes \mathbb{R}$ by $b \mapsto g \cdot b$.

Then $\delta(G)=\left(\bigsqcup_{S} \delta(S)\right) / \sim$. We will denote the equivalence class of $b$ in $\delta(G)$ by $[b]_{G}$.

The association of $\&(G)$ to $G$ is a functor from the category of connected reductive groups over $k$ with monomorphisms as morphisms, to the category of sets with injections as morphisms.

(3.3) An alternative description For our purpose, it is convenient to give a variant of the above description of $\delta(G)$.

Given $b \in \delta(S)$ as above, there is a smallest subtorus $T_{b}$ of $S$ such that $b \in \delta\left(T_{b}\right)$. The functorial inclusions $\delta\left(T_{b}\right) \hookrightarrow \delta(S) \hookrightarrow \delta(G)$ send $[b]_{T_{b}}$ to $[b]_{S}$ to $[b]_{G}$. For any $k$-split torus $T$, define $\delta^{\sharp}(T)$ to be the subset of $\delta(T)$ consisting of those $b$ such that $T_{b}=T$. Then $\delta(G)$ is the union of $\delta^{\sharp}(T)$, as $T$ ranges over all $k$-split subtori of $G$. Here, we identify $\delta^{\sharp}(T)$ with its image in $\delta(G)$ by functoriality.

Lemma Suppose that $T, T^{\prime}$ are k-split subtori of $G$ and $b \in 8^{\sharp}(T), b^{\prime} \in$ $s^{\sharp}\left(T^{\prime}\right)$. Then $[b]_{G}=\left[b^{\prime}\right]_{G}$ if and only if there exists $u \in R_{\mathrm{u}}(P(b))(k)$ such that $u T u^{-1}=T^{\prime}$ and $u \cdot b=b^{\prime}$. Moreover, such an element $u$ is unique.

Proof. Let $S$ be a maximal $k$-split torus of $G$ containing $T$, and let $\left\{U_{a}\right\}_{a \in \Phi(G, S)}$ be the root subgroups of $G$ relative to $S$. Then $P(b)$ is generated by $Z_{G}(S)$ and those $U_{a}$ with $\langle a, b\rangle \geqslant 0$. Moreover, $P(b)=L(b) \ltimes R_{\mathrm{u}}(P(b))$, where $L(b)$ is generated by $Z_{G}(S)$ and the $U_{a}$ for $a$ satisfying $\langle a, b\rangle=0$. It is easy to see that $P(b)$ and $L(b)$ do not depend on the choice of $S$. In fact, $G(k)$ acts on the disjoint union $\bigsqcup_{T} \mathcal{F}^{\sharp}(T)$ and $L(b)(k)$ is simply the fixer of $b$.

Similarly, let $S^{\prime}$ be a maximal $k$-split torus of $G$ containing $T^{\prime}$. If $[b]_{G}=\left[b^{\prime}\right]_{G}$, by definition, there exists $g \in P(b)(k)$ such that $g S g^{-1}=S^{\prime}$ and $g \cdot b=b^{\prime}$. It is then clear that $g T g^{-1}=T^{\prime}$. Write $g=u l$ with $u \in R_{\mathrm{u}}(P(b))(k)$ and $l \in L(b)(k)$. Then it is easy to see that $l T l^{-1}=T$ and $l \cdot b=b$. Therefore, $u T u^{-1}=T^{\prime}$ and $u \cdot b=b^{\prime}$. The converse is also clear.

The uniqueness of $u$ follows from the fact that the fixer of $b$ is $L(b)(k)$ and $L(b) \cap R_{\mathrm{u}}(P(b))=\{1\}$.

(3.4) Proposition If the order of $F$ is prime to $p$, then $s(G)=\varsigma(H)^{F}$.

Proof. Suppose that $y \in \delta(H)^{F}$. Represent $y$ by $b \in \delta^{\sharp}(T)$. For each $s \in F$, since $s . y=y$, there exists a unique $u_{s} \in R_{\mathrm{u}}(P(b))(k)$ such that $s . T=u_{s}^{-1} T u_{s}, s . b=u_{s}^{-1} \cdot b$. 
It follows that $s \mapsto u_{s}$ is a 1-cocycle with values in the $p$-group $R_{\mathrm{u}}(P(b))(k)$, hence is a coboundary: $u_{s}=v^{-1}($ s.v $)$ for some $v \in$ $R_{\mathrm{u}}(P(b))(k)$. Here, by a $p$-group we mean a nilpotent group $M$ such that $M_{i} / M_{i+1}$ is killed by a power of $p$ for each $i \geqslant 0$, where $\left\{M_{i}\right\}$ denotes the lower central series of $M$. Replacing $(T, b)$ by $\left(v T v^{-1}, v \cdot b\right)$, we may assume that $T$ is $F$-stable and $u_{s}=1$ for all $s \in F$.

Represent $b \in \delta(T)$ by $\tilde{b} \in X_{*}(T) \otimes \mathbb{R} \backslash\{0\}$. Then for each $s \in F$, there is a unique $r_{s}>0$ such that $s . \tilde{b}=r_{s} \tilde{b}$. It follows that $s \mapsto r_{s}$ is a homomorphism from $F$ into $\mathbb{R}_{>0}$, hence $r_{s}=1$ for all $s \in F$. This implies that $\tilde{b} \in X_{*}(T)^{F} \otimes \mathbb{R}=X_{*}\left(T^{F}\right) \otimes \mathbb{R}$ and hence $T^{F}=T$. Therefore, $T \subset G$ and $b$ represents a point on $\delta(G) \subset \delta(H)$. The proposition is proved.

(3.5) Remark When $F$ is cyclic, generated by a semisimple element of order prime to $p$, this proposition reduces to a special case of [CLT, Proposition 5.1]. When $F$ is of order 2, this proposition has also been observed independently by Kim and Moy [KM].

Again, if the cardinality of $F$ is divisible by $p, \delta(H)^{F}$ may be strictly bigger than $\delta(G)$. See [CLT, Proposition 8.4].

\section{References}

[BT1] F. Bruhat, J. Tits, Groupes réductifs sur un corps local I. Publ. Math. I.H.E.S. 41 (1972), 5-251

[BT2] F. Bruhat, J. Tits, Groupes réductifs sur un corps local II. Publ. Math. I.H.E.S. 60 (1984), 197-376

[BT3] F. Bruhat, J. Tits, Schémas en groupes et immeubles des groupes classiques sur un corps local. Bulletin Soc. Math. France 112 (1984), 259-301

[BT4] F. Bruhat, J. Tits, Schémas en groupes et immeubles des groupes classiques sur un corps local II: groupes unitaires. Bulletin Soc. Math. France 115 (1987), 141-195

[CLT] C.W. Curtis, G.I. Lehrer, J. Tits, Spherical buildings and the character of the Steinberg representation. Invent. math. 58 (1980), 201-210

[E] B. Edixhoven, Néron models and tame ramification. Compositio Math. 81 (1992), 291-306

[GY] W.T. Gan, J.-K. Yu, Schémas en groupes et immeubles des groupes exceptionnels sur un corps local, Première partie: Le groupe $G_{2}$. Preprint (2000)

[KM] J.-L. Kim, A. Moy, Involutions, classical groups and buildings. J. Algebra 242 (2001), 495-515

[L] E. Landvogt, Some functorial properties of the Bruhat-Tits building. J. Reine Angew. Math. 518 (2000), 213-241

[MP] A. Moy, G. Prasad, Jacquet functors and unrefined minimal $K$-types. Comment. Math. Helvetici 71 (1996), 96-121

[P] G. Prasad, Galois-fixed points in the Bruhat-Tits building of a reductive group. Bulletin Soc. Math. France 129 (2001), 169-174

[PR] V. Platonov, A. Rapinchuk, Algebraic groups and number theory. Academic Press (1994)

[R] R.W. Richardson, Affine coset spaces of reductive algebraic groups. Bull. London Math. Soc. 9 (1977), 38-41

[S1] R. Steinberg, Endomorphisms of linear algebraic groups. Memoirs of the Amer. Math. Soc. 80 (1968)

[S2] R. Steinberg, Torsion in reductive groups. Adv. in Math. 15 (1975), 63-92

[T] J. Tits, Reductive groups over local fields. Automorphic forms, representations, and $L$-functions (A. Borel, W. Casselman, Eds.), v.1., 29-69 (1977) 